\title{
Tip-enhanced Raman spectroscopic imaging shows segregation within binary self-assembled thiol monolayers at ambient conditions
}

\author{
Wan-Ing Lin $^{1} \cdot$ Feng Shao $^{1} \cdot$ Bruno Stephanidis $^{1} \cdot$ Renato Zenobi $^{1}$
}

Received: 11 April 2015 /Revised: 28 May 2015 / Accepted: 2 June 2015 / Published online: 28 June 2015

(C) Springer-Verlag Berlin Heidelberg 2015

\begin{abstract}
Phase segregation of coadsorbed thiol molecules on a gold surface was investigated with nanoscale chemical imaging using tip-enhanced Raman spectroscopy (TERS). Samples were prepared using mixed solutions containing thiophenol $(\mathrm{PhS})$ and an oligomeric phenylene-ethynylene (OPE) thiol, with 10:1, 2:1, and 1:1 molar ratios. Phase segregation into domains with sizes from $\approx 30$ to $240 \mathrm{~nm}$ is observed with these molar ratios. A comparison of TERS images with different pixel sizes indicates that a pixel size bigger than $15 \mathrm{~nm}$ is not reliable in defining nanodomains, because of undersampling. In this study, the formation of nanodomains was clearly evident based on the molecular fingerprints provided by TERS, while ambient scanning tunneling microscopy (STM) was not capable of discerning individual domains via their apparent height difference. TERS therefore allows to image nanodomains in binary self-assembled monolayers, which are invisible to methods solely relying on topographic or electron density characteristics of self-assembled monolayers. Moreover, TERS mapping provides statistical data to describe the distribution of molecules on the sample surface in a well-defined manner. Peak ratio histograms of selected TERS signals from samples prepared with different mixing ratios give a better understanding of the adsorption preference
\end{abstract}

Published in the topical collection Nanospectroscopy with guest editor Mustafa Culha.

Electronic supplementary material The online version of this article (doi:10.1007/s00216-015-8840-x) contains supplementary material, which is available to authorized users.

Renato Zenobi

zenobi@org.chem.ethz.ch

1 Department of Chemistry and Applied Biosciences, ETH Zurich, 8093 Zurich, Switzerland of the thiols studied, and the relationship of their mixing ratio in solution and adsorbed on the surface.

Keywords Thiols $\cdot$ Raman spectroscopy $\cdot$ Imaging . Self-assembled monolayers · TERS $\cdot$ Segregation

\section{Introduction}

Self-assembled monolayers (SAMs) on gold or silver surfaces are widely employed to generate tailored surfaces, for example, to influence surface wetting properties $[1,2]$ and change rates of charge transport [3] and for chemical functionalization [4]. By utilizing a variety of thiols with different end groups and variable chain length, many properties on the surface can be custom-designed. Binary SAMs formed on a surface permit tuning of electronic properties such as the work function $[5,6]$. If binary SAMs form domains, an alternating order of different thiol monolayers with distinct chemical properties on a surface allows interesting applications, for example, for dual-affinity biosensors $[7,8]$. Phase segregation of twocomponent thiol monolayers has been observed for functionalized and unfunctionalized thiols [9-11], and was found to occur only when certain mixing ratios were used [12]. For example, in the case of mixing 4-aminothiophenol (4-ATP) and $n$-octadecanthiol (ODT), a clear phase segregation occurred for a $60 \%$ 4-ATP molar ratio, with 10 to $100 \mathrm{~nm} \mathrm{4-}$ ATP islands. If the molar fraction of 4-ATP increased above $60 \%$, homogeneous mixing occurred. However, domain formation in other mixed thiol SAMs is still relatively poorly understood. This, in part, is because there are many different thiols that are being used for such experiments, all exhibiting different adsorption behavior and intermolecular forces. Moreover, methods for their investigation on the nanometer length scale are scarce and typically lack chemical specificity. 
Mixed thiol SAMs are known to be more disordered compared to pure alkanethiolate monolayers, where a crystalline structure is often observed $[13,14]$. To investigate multifunctional surface structures, for example at molecular recognition sites of sensors or for artificial receptors, it is required to visualize them on a nanometer length scale [12]. Some research groups have attempted to use conventional scanning probe microscopy (scanning tunneling microscopy, STM, or atomic force microscopy, AFM) to distinguish domains but failed to discern different chemisorbed molecules based on their height $[12,15]$, although friction force AFM did reveal contrast [12]. Characterizing the molecular structure of SAMs is possible with high-resolution STM imaging at ambient conditions [16]; however, even in a pure SAM, many packing patterns could be observed [17], which means that the height measured by SPM can hardly identify the compounds in a mixed SAM. Additionally, the tunneling current in STM reflects not only the tip-sample distance but also the local electronic structure of the surface, which will require further complex data processing to reveal the domains [18]. The fact that there is hardly any literature available on characterizing mixed SAMs by SPM methods thus implies severe technical difficulties. Methods other than SPM including spectroscopic techniques $[19,20]$, contact angle measurements [21], and mass spectrometry [22] have also been applied to characterize SAMs. Global spectroscopic methods provide precise structural/ molecular information; however, they are limited in spatial resolution. Therefore, a method to analyze complex SAMs with chemical specificity and nanoscale spatial resolution is needed.

Tip-enhanced Raman spectroscopy (TERS) is a combined SPM and Raman technique, which allows to simultaneously acquire spectroscopic fingerprint information and topographical features with $\approx 9-15 \mathrm{~nm}$ spatial resolution [23-25] at ambient conditions. Invented in 2000 [26-28], this near-field optical technique breaks the optical diffraction limit by employing a nanoantenna as a Raman signal amplifier. TERS allows one to obtain signals from a small number of probed molecules, rendering monolayers $[15,29,30]$ and sometimes even single molecules on surfaces spectroscopically visible [31-33]. Early work using TERS to study SAMs was reported by Picardi [34]. An important finding was that the tunneling parameters in STM-TERS can strongly influence the enhanced Raman signal intensities. Chemical imaging by means of TERS was also used to identify the distribution of two patterned isomeric thiols on a gold surface, where STM cannot distinguish them but spectral imaging does [15]. TERS has also recently been used to study binary SAMs on a gold substrate $[35,36]$. Picardi et al. [35] showed that the subsequent exchange of molecules within a SAM by a second thiol occurred preferentially at grain boundaries of the gold substrate, and Horimoto et al. [36] showed a gradual change of the molecular composition of the original SAM with increasing immersion time in the second thiol solution.

There are two methods to prepare binary SAMs: (i) coadsorption and (ii) partial exchange of molecules in a pure SAM. In the present study, binary SAMs produced by coadsorption are studied by TERS for the first time. Whether the molecules chosen for this study form domains is still unexplored. If binary SAMs indeed form domains, TERS should potentially be able to visualize this domain formation. Domains have been reported with sizes ranging from $10 \mathrm{~nm}$ to conjugated patches (i.e., stripelike domains) $[37,38]$. With an appropriately chosen pixel size, it should be possible to see nanoscale domains with TERS. In addition, based on TERS mapping on a binary SAM and acquisition of spectra at different locations on the sample, statistical data evaluation is in principle possible to describe the nanoscale composition of the monolayers. Performing point measurements rather than mapping, for example, does not answer the question whether the molar ratio of thiols in solution is reflected in the molar ratio in the SAM.

Here, we demonstrate full spectroscopic mapping of binary SAMs, with a pixel size down to $2 \mathrm{~nm}$. An oligomeric phenylene-ethynylene (OPE) was chosen for this study since conjugated phenylene-ethynylene oligomers are of interest for molecular electronics and have tunable functionality. We chose $S$-[4-[2-[4-(2-phenylethynyl)phenyl]ethynyl]phenyl] thioacetate to represent an OPE system and produced mixed monolayers with thiophenol $(\mathrm{PhS})$. The mixture of these two thiols is appropriate for TERS studies because they are both good Raman scatterers and have similar polarities but distinctly different physical heights; if they form domains, the only prominent intermolecular forces, i.e., van der Waals forces, govern the molecular packing at ambient conditions.

\section{Experimental}

Setup TERS measurements were carried out on a commercial combined STM/Raman microscope (NTEGRA spectra Upright, NT-MDT, Zelenograd, Russia) as described previously [39]. A HeNe laser (632.8 nm) was utilized for illumination, with a typical laser power of $95 \mu \mathrm{W}$ on the sample stage. The acquisition time was chosen between 1 and 3 s. STM images were recorded with the same tip before or after TERS mapping. STM measurements were performed in constant current mode with a set point of $0.1 \mathrm{nA}$ and a bias voltage of $0.1 \mathrm{~V}$. High-resolution STM images with a smaller scan range (less than $500 \times 500 \mathrm{~nm}^{2}$ ) were recorded with a tabletop STM (Easyscan, Nanosurf, Liestal, Switzerland). 
TERS tips Silver wires (diameter $0.25 \mathrm{~mm}, 99.99 \% \mathrm{pu}-$ rity, Alfa Aesar, Germany) were electrochemically etched at a voltage of $10 \mathrm{~V}$ in a 1:4 $(\mathrm{v} / \mathrm{v})$ mixture of perchloric acid (70\%, Sigma-Aldrich, Buchs, Switzerland) and ethanol (p.a., Sigma-Aldrich, Buchs, Switzerland), similar to the procedure previously described by Blum et al. [40]. The etched tips were rinsed with ethanol and water and checked for their sharpness with a Nikon $360 \times$ stereomicroscope. The tip was afterwards mounted on the tip holder of the NT-MDT system, and stable tunneling feedback was established. The focused laser beam was scanned over the tip while Raman spectra were collected at every position. Details of this procedure ("hot spot localization") were given previously [25, 41].

Samples Thioacetic acid $S$-[4-[4-(phenylethynyl)phenyl]ethynyl]benzene-thiol ester (as an OPE) (SigmaAldrich) and thiophenol (Acros, USA) were purchased. Deprotection of OPE was accomplished by hydrolysis of thioacetate in ethanol $(0.5 \mathrm{mM})$ by adding $\mathrm{NaOH}$ to the solution $(10 \mu \mathrm{L}, 1 \mathrm{~N})$ as a hydrolyzing agent. Thiophenol $(\mathrm{PhS})$ was prepared as $10 \mathrm{mM}$ solution in ethanol. Au(111) on mica (4×4 mm, \#20020020, from Phasis, Geneva, Switzerland) was immersed in the ethanolic thiol solution for $24 \mathrm{~h}$ at room temperature. We used three different mixing ratios of $\mathrm{PhS}$ and OPE (corresponding to the molar ratio of the molecules in solution), i.e., $\mathrm{PhS}: \mathrm{OPE}=10: 1,2: 1$, and 1:1, with the intention of obtaining binary SAMs with different domain sizes [42]. The concentration of $\mathrm{PhS}$ was chosen to be higher than that of OPE because the latter is the stronger Raman scatterer [43].

Data processing Spectra were processed in MATLAB (version R2014b, MathWorks, Natick, MA, USA). The procedure included background subtraction followed by smoothing (moving average and a robust version of locally weighted scatterplot smoothing, LOESS). In order to investigate the spatial distribution of the two thiol molecules, the intensity ratio of the peaks at $1129 \mathrm{~cm}^{-1}$ ("peak 1") and $1077 \mathrm{~cm}^{-1}$ ("peak 2") was calculated at every position of the map. Only pixels with a signal-to-noise ratio higher than 3 for either of the evaluated peaks were taken into account. Pixels containing just noise in the map are marked as such. We could also have chosen the peak of the triple bond band as an OPE marker, which appears only in the OPE spectrum, but peaks 1 and 2 are much closer to each other in the spectrum, less affected by variations in background and any possible wavelength dependence of the enhancement, which should be a more reliable measure of the local composition. Besides the ratio map of peak $1 /$ peak 2 , the absolute intensity of the peak at $2214 \mathrm{~cm}^{-1}$, the signal due to the triple bond, is also shown for comparison.

STM images were processed with Gwyddion (a modular program for SPM data analysis [http://gwyddion.net/]), and
2D-plane subtraction (slope correction) and line mean corrections were carried out.

\section{Results and discussion}

An atomically flat gold surface was used for this study. An STM image of a bare gold substrate is shown in the Electronic Supplementary Material (ESM) in Fig. S1. The measured step height is $0.2 \mathrm{~nm}$ between adjacent gold terraces, close to the expected height of an atomic step on Au [44]. An OPE SAM on gold was then investigated with STM and TERS; the STM topography shows that imaging the structure of single thiol SAMs at ambient conditions reveals little information (ESM Fig. S2).

A high-resolution STM scan of a mixed SAM sample prepared with a $10: 1 / \mathrm{PhS}: \mathrm{OPE}$ molar ratio in solution is shown in Fig. 1a. The corresponding height profile (Fig. 1b) exhibits a low noise level (rms $0.0064 \mathrm{~nm}$ ). The maximum expected height difference of the two thiols is $1.6 \mathrm{~nm}$, but no areas with a height difference of that order are visible in the profile. One reason for this may be that $\mathrm{PhS}$ and $\mathrm{OPE}$ exhibit different adsorption angles on $\mathrm{Au}(111)$ such that even if domains form, no height difference is evident. In other words, it can be tricky to characterize mixed adsorbates by STM alone. Consequently, TERS maps were acquired to study the phase segregation of binary SAMs on an Au surface.

The thiols chosen, OPE and $\mathrm{PhS}$, have quite different Raman spectra. TERS spectra of pure OPE and PhS are shown in Fig. 2. The peaks at 1002, 1023, 1077, and $1580 \mathrm{~cm}^{-1}$ are signals due to aromatic ring vibrations. Whereas the peaks at 1002,1077 , and $1580 \mathrm{~cm}^{-1}$ are visible both in OPE and $\mathrm{PhS}$, the peak at $1129 \mathrm{~cm}^{-1}$, assigned to a $v(\mathrm{C}-\mathrm{H})$ in-plane mode [45], is only observed and particularly strong in the OPE spectrum; this mode is pronounced only in molecules with three phenyl rings [46, 47]; hence, it can be used as a marker band for OPE when studying the distribution of OPE and $\mathrm{PhS}$ on the surface. While the peak at $1023 \mathrm{~cm}^{-1}$ is exclusively observed in PhS spectra, its intensity was too close to the noise level in many of the spectra. This ruled out its use as a marker band for $\mathrm{PhS}$. In order to investigate the spatial distribution of these two thiol molecules, the intensity ratio of the peaks at 1129 and $1077 \mathrm{~cm}^{-1}$ was therefore calculated at every position of the TERS maps.

A TERS map of a pure OPE sample with 4092 points was measured and the peak ratio was plotted (Fig. 3a). The histogram in Fig. 3b shows that the ratio of the two marker bands $\left(1129 / 1077 \mathrm{~cm}^{-1}\right)$ exhibits a Gaussian distribution, with a mean value of 2.51 and a standard deviation of 0.47 . The variation of this ratio may result from the angle of the molecules relative to the surface, which directly affects the orientation of the Raman scattering tensor. OPE is the larger molecule and can thus sample a larger range of distances to the 


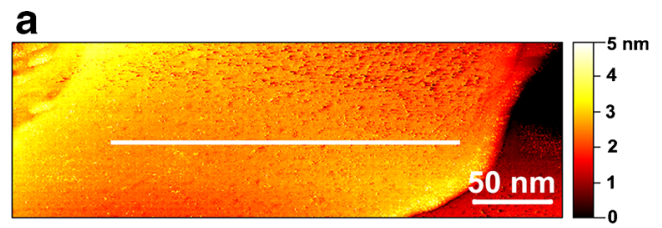

\section{b}

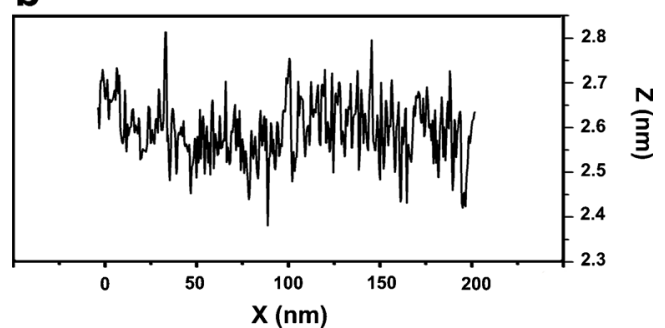

Fig. 1 a STM image (area $340 \times 110 \mathrm{~nm}^{2}$ ) of a PhS/OPE binary SAM on $\mathrm{Au}(111)$. b As can be seen in the cross-sectional profile, the measurement fails to discern molecular domains via their expected maximum height difference of $1.6 \mathrm{~nm}$

surface if it assumes different orientations [47]. Additionally, since the peaks at 1129 and $1077 \mathrm{~cm}^{-1}$ are both $\mathrm{CH}$ in-plane bending modes [45], they will only be excited at the same time, which eliminates another possible systematic error if the intensity ratio of these two peaks is used [48]. From this data, it can be concluded that a pure OPE TERS spectrum exhibits a peak ratio between 2.04 and 2.98 with $68 \%$ probability and between a ratio of 1.57 to 3.44 with $95 \%$ probability. The peak at $1129 \mathrm{~cm}^{-1}$ is only observable in the OPE spectrum; hence, if a spectrum of a binary SAM has a peak ratio significantly above 2 , such a pixel contains mostly OPE molecules. In contrast, if the $1077 \mathrm{~cm}^{-1}$ peak becomes stronger, which means that there is a large contribution from $\mathrm{PhS}$ molecules, the peak ratio will drop to less than 2. Taking somewhat arbitrary thresholds for the peak ratios, we consider pixels with a ratio between 1 and 2 to contain a considerable amount of $\mathrm{PhS}$, and predominantly $\mathrm{PhS}$ molecules if the ratio is between 0.5 and 1 , while pixels with a peak ratio of less than

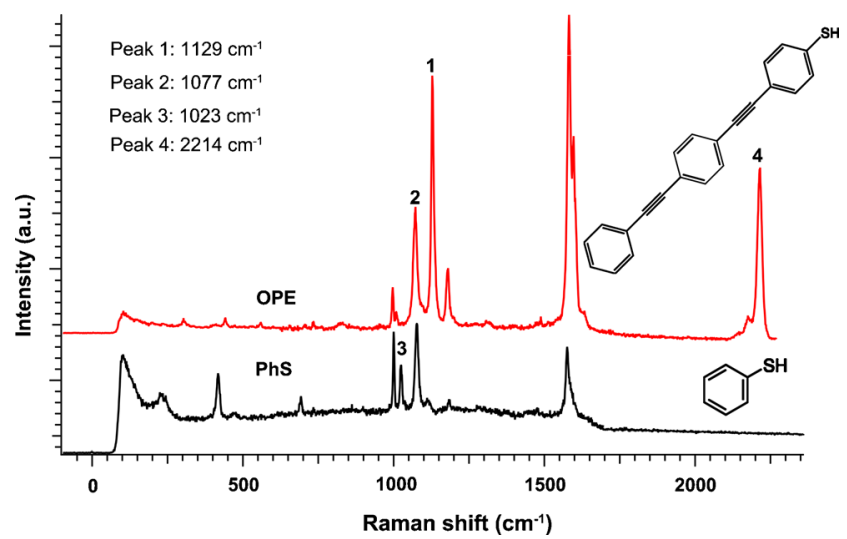

Fig. 2 TERS spectra of pure thiols forming SAMs on a gold surface. The black spectrum is from a SAM of thiophenol $(\mathrm{PhS})$ and the red spectrum from a SAM of OPE on gold a

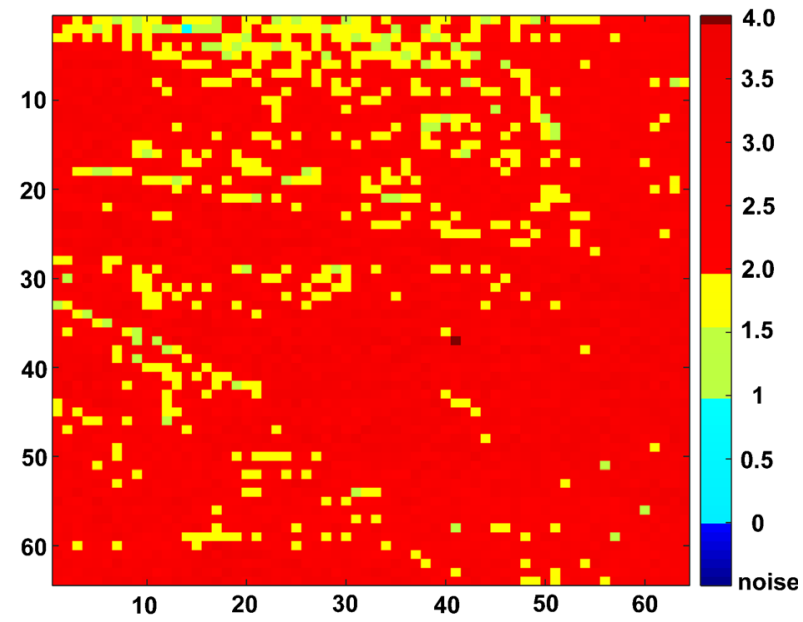

b

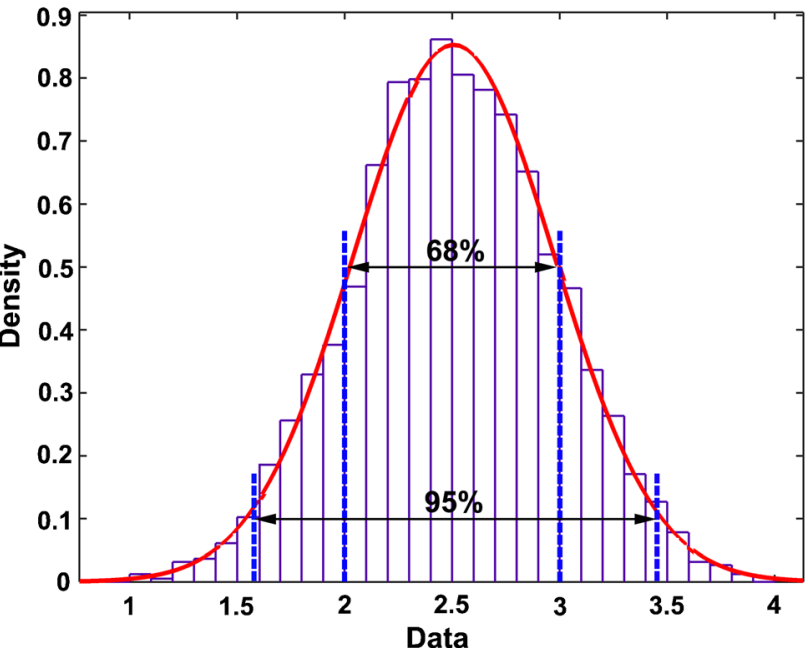

Fig. 3 a A TERS peak ratio map of a pure OPE SAM (showing the intensity ratio of peak $1129 \mathrm{~cm}^{-1} /$ peak $1077 \mathrm{~cm}^{-1}$ ) measured over a $304 \mathrm{~nm} \times 304 \mathrm{~nm}$ area with $64 \times 64$ points; the color coding of the peak ratio map in all figures is the same. b A histogram of peak ratios in 4096 points fitted with normal distribution, showing that $68 \%$ of the data is in the range of $2-3$ ratio value

0.5 imply essentially a pure $\mathrm{PhS}$ island. Employing the peak ratios to compare the amount of components in a mixture has been reported before [30, 36, 49, 50]. Accordingly, all TERS maps below are shown with the false color scale as depicted in Fig. 3a, with red representing pure OPE. Nevertheless, we have to caution that it is difficult to determine accurate surface coverages of the two thiols from peak ratios by TERS.

Figure $4 \mathrm{a}$ shows a TERS map $\left(512 \times 512 \mathrm{~nm}^{2}\right.$ and $32 \times$ 32 pixels) of a binary SAM prepared with a 1:1 ratio. Large domains of OPE and $\mathrm{PhS}$ can be easily seen from the map, i.e., phase segregation clearly takes place. $\mathrm{PhS}$ is, for example, dominant in the blue area in the bottom right of the map. The size of the largest $\mathrm{PhS}$ domain is bigger than $64 \times$ $240 \mathrm{~nm}^{2}$, and additionally smaller islands with a length of $48 \mathrm{~nm}$ (3 pixels) are visible. OPE also shows two pronounced 
Fig. 4 a TERS peak ratio map of a binary SAM (with a 1:1 ratio), $512 \times 512 \mathrm{~nm}^{2}$ and $32 \times 32$ pixels $(16 \mathrm{~nm} /$ pixel) was measured. b An intensity map of the $2214 \mathrm{~cm}^{-1}$ peak (triple bond). $\mathbf{c}$ The STM image acquired in the same area after the TERS measurement. Several domains are included but not visible as the height differences in the STM data. d Cross-sectional line profile showing the variation in height across a PhS island. Note: the 100-nm scale bar applies to all maps in $\mathbf{a}-\mathbf{c}$
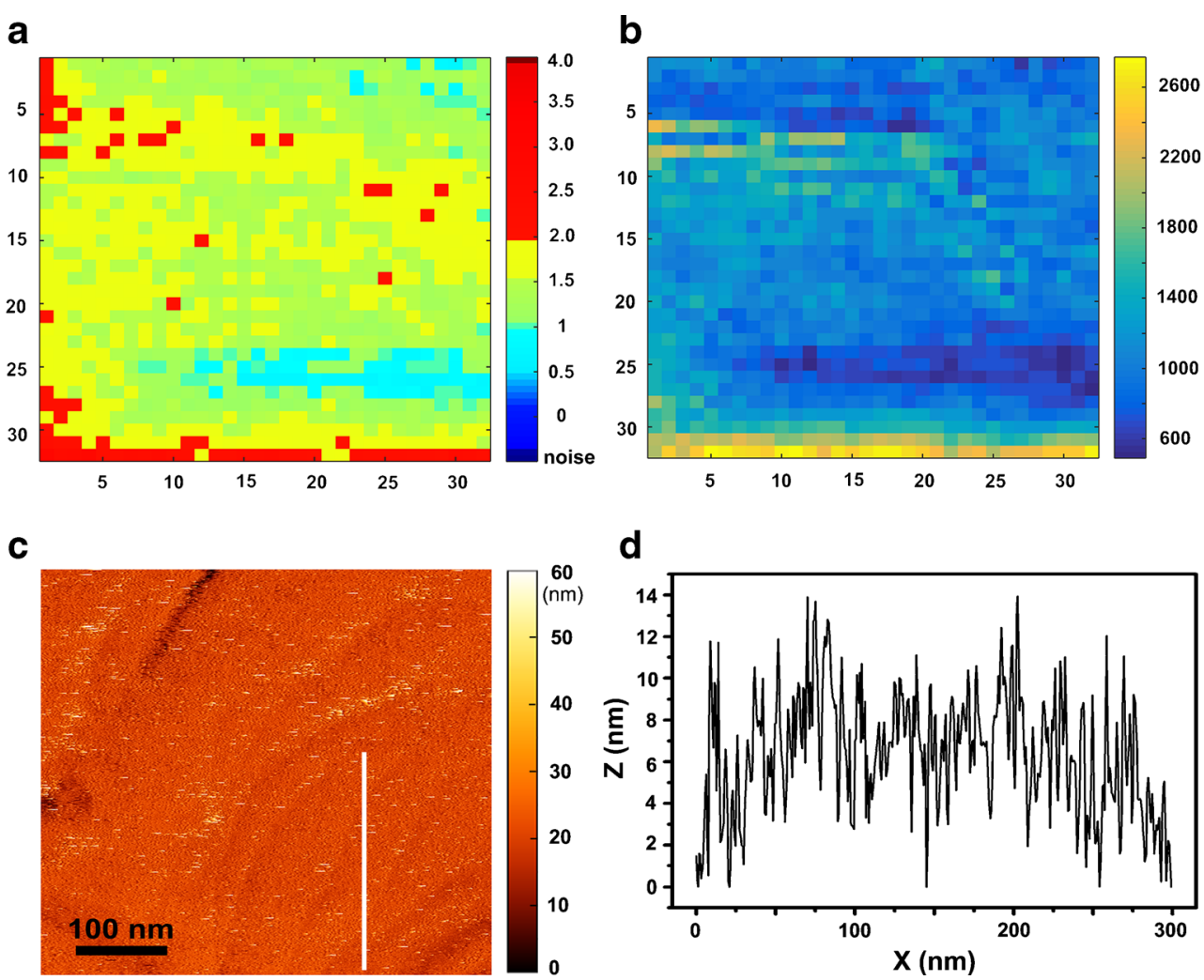

\section{d}

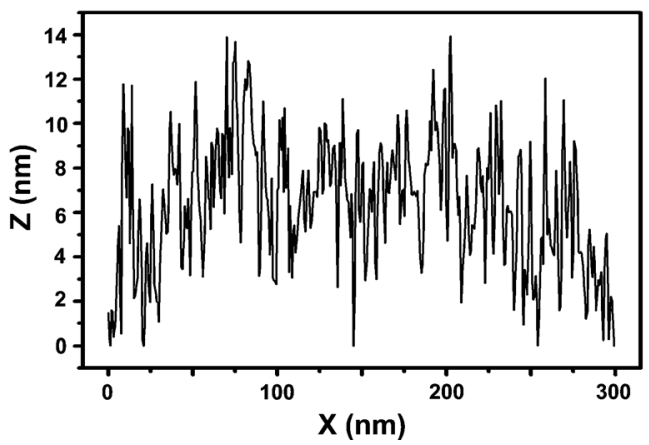

domains colored in red on the map with few isolated pixels dispersed in the yellow area, where $\mathrm{PhS}$ is interfacially present but OPE is dominant. This shows that the determination of domains is not an artifact from the feedback (the STM was operated with a fast X scan and a slow Y scan). Also, variations in the distance between the tip and the surface can have a significant influence on the signal intensity [35] but should have no effect on peak ratios.

A corresponding map of the absolute intensity of the triple bond is also shown in Fig. 4b. The area where OPE is abundant (bright yellow in Fig. 4b) shows a high triple bond intensity, whereas in the area where $\mathrm{PhS}$ domains are located (peak ratio less than 1), the triple bond signal intensity is low (dark blue), which coincides very well with the ratio map of the two marker bands. The absolute intensity distribution map of the peak at $2214 \mathrm{~cm}^{-1}$ shows features that are consistent with the peak ratio map. We hypothesize that this is because the modes corresponding to the peaks at 1129 and $2214 \mathrm{~cm}^{-1}$ are of the same symmetry [48]. This supports the method of using peak ratios of the same symmetry to compare the amount of two thiol molecules.

An STM image is shown in Fig. $4 \mathrm{c}$ with $512 \times 512$ points $\left(512 \times 512 \mathrm{~nm}^{2}\right)$ recorded right after the TERS imaging experiment. The STM image does not show any clear height differences. Atomic steps of the underlying gold substrate seem to be observable, but domains in the binary SAM were not discernable based on height differences. A cross-sectional line profile is shown in Fig. 4d (spikes were removed), which in this case shows an rms height variation of $2.85 \mathrm{~nm}$. This again illustrates the difficulty and inconvenience to define domains by STM height alone.

Figure 5 a shows a $3 \times 3 \mu \mathrm{m}^{2}$ STM topographic image of a binary PhS/OPE SAM on Au(111) prepared with a 10:1 molar ratio in solution. Several large gold grains $(500-1000 \mathrm{~nm})$ with rather smooth surfaces can be seen. The TERS map in Fig. $5 \mathrm{~b}$ was measured over the whole area using $32 \times 32$ points with a $96 \mathrm{~nm}$ pixel size. Phase segregation can again be observed in the map. Two domains with a peak ratio $>2$ can be discerned, with a size around $0.5 \times 1$ and $1 \times 1 \mu^{2}$. Compared to the STM image, these two OPE-rich domains seem to cover the surface of the gold grain underneath. In the upper part of the image, there is a large area with a peak ratio below 0.5 (dark blue; the peak at $1129 \mathrm{~cm}^{-1}$ has very low intensity), indicating a domain of pure $\mathrm{PhS}$ with a dimension of $\approx 2.5 \times$ $0.5 \mu^{2}$. When zooming into an area of this map (red rectangle), an STM image was first recorded (Fig. 5c), followed by a TERS map (Fig. 5d). However, the segregation distribution does not reproduce the image in Fig. 5a. The domain size of OPE now appears to be $\approx 290 \times 580 \mathrm{~nm}^{2}$.

Domains of PhS and OPE are easy to distinguish in these three TERS maps, which reveal two-component SAMs to be segregated instead of intermixed. An STM image acquired during the TERS map (Fig. 5d) is also available (see ESM Fig. S3a) to prove that sample drift is insignificant. At first 
Fig. 5 a STM topography image $\left(3 \times 3 \mu \mathrm{m}^{2}\right)$. b A TERS peak ratio map of a binary SAM (with a 10:1 ratio), $32 \times 32$ pixels on the same area as $96 \mathrm{~nm} /$ pixel. c Zooming into the red rectangle, an STM image was first acquired with $1.5 \mu \mathrm{m} \times 1.0 \mu \mathrm{m}$ before TERS. d The TERS peak ratio map corresponds to the area in $\mathbf{c}$, $48 \mathrm{~nm} /$ pixel. e An STM image by zooming into the red square in c. f The TERS peak ratio map corresponds to the area in $\mathbf{e}$, $16 \mathrm{~nm} /$ pixel. Pixels in brown and dark blue have signals at the noise level

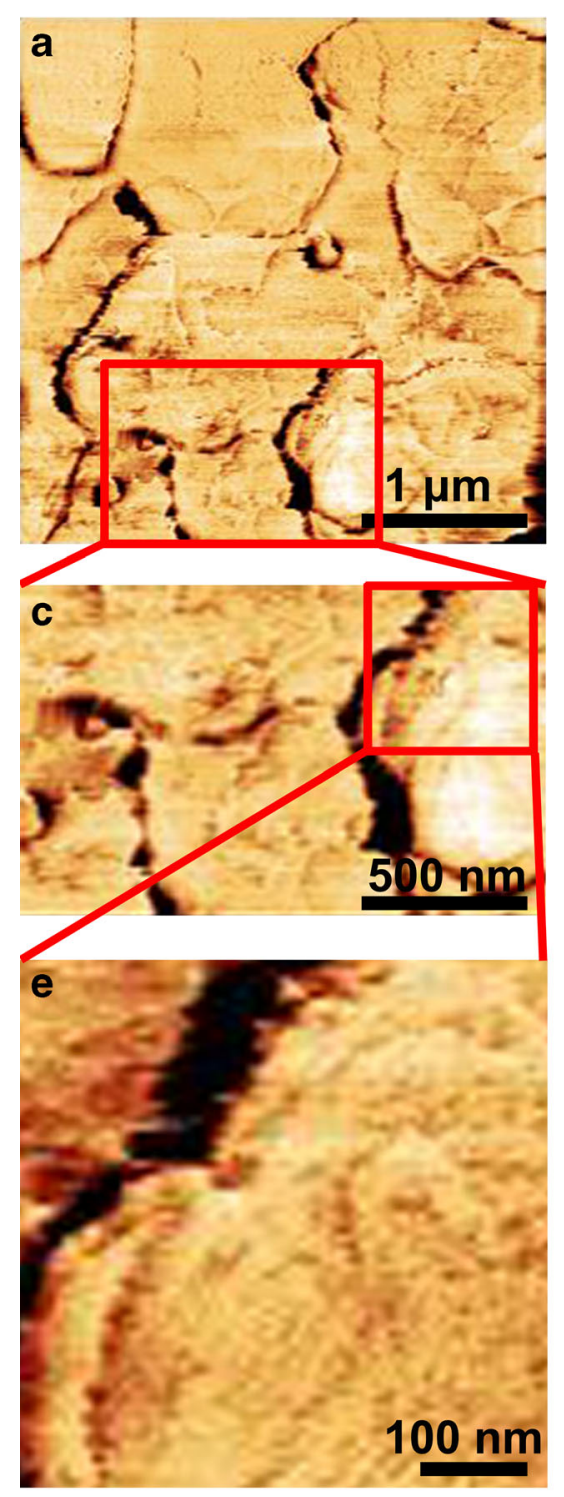

glance, it seems strange that the size of the molecular domains is reduced in TERS maps recorded with a smaller pixel size. This phenomenon can be rationalized by the TERS

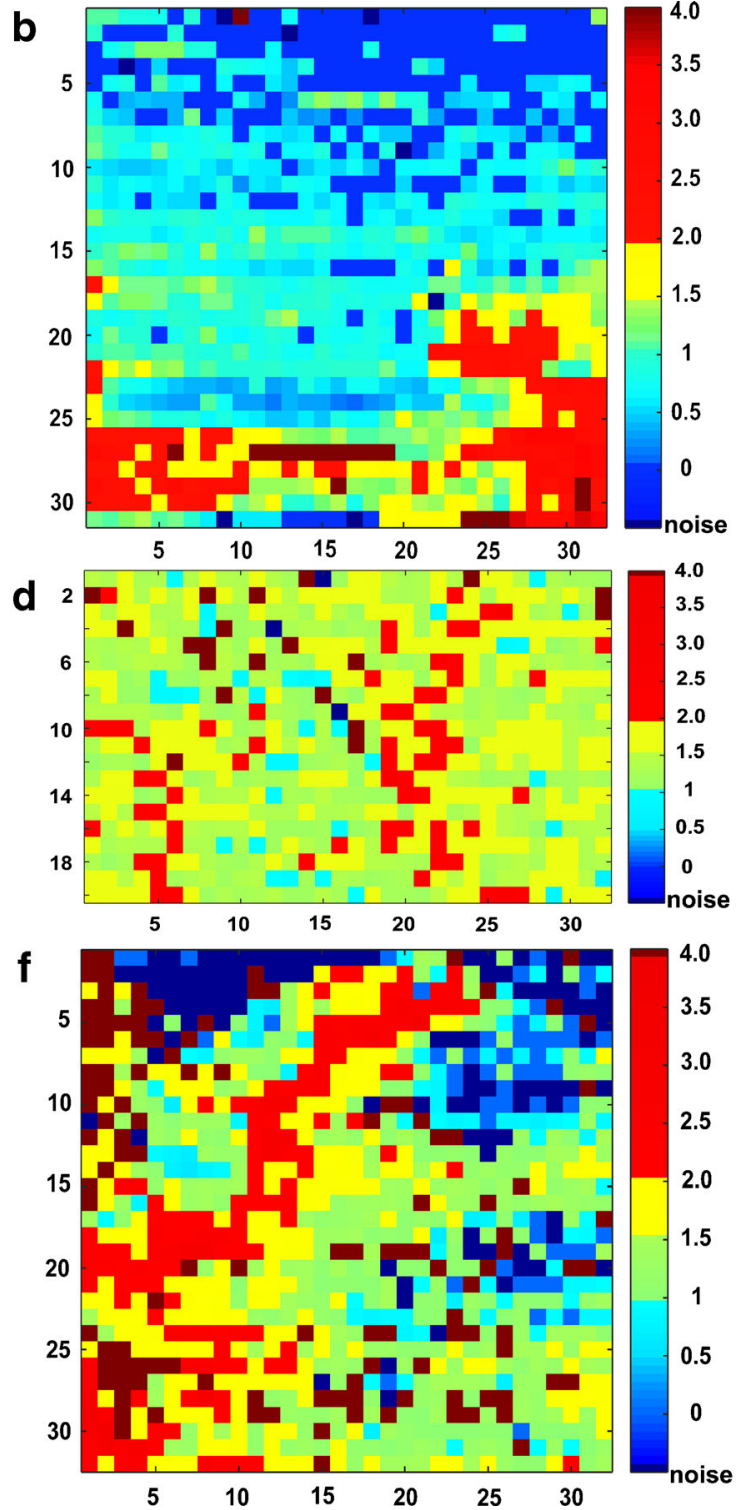

a

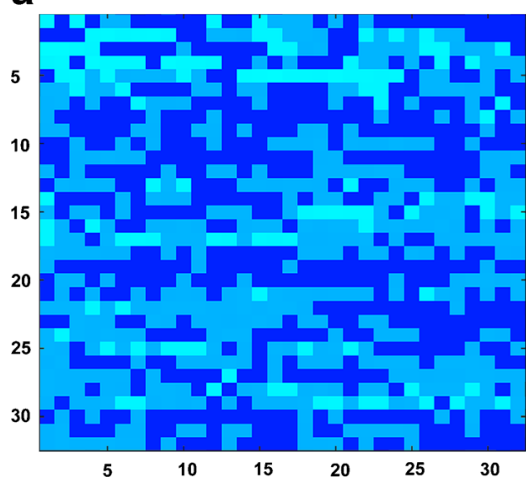

b

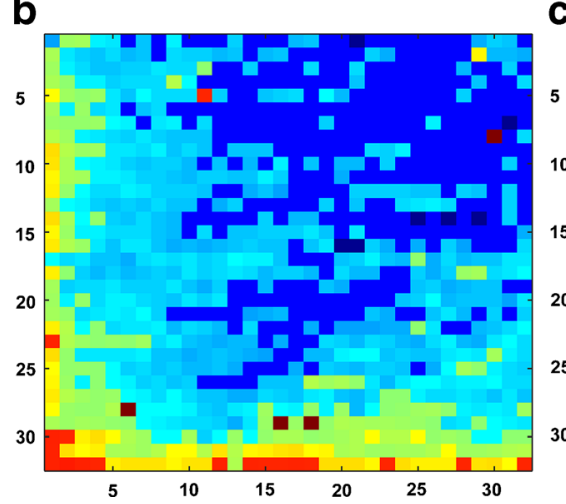

C

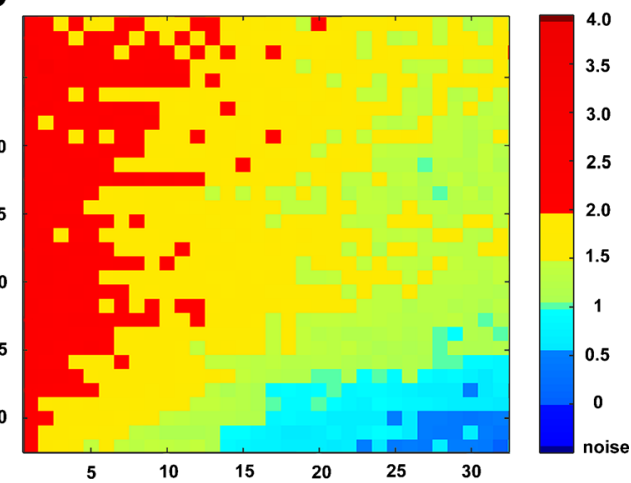

Fig. 6 TERS peak ratio maps of binary SAMs measured with $8 \mathrm{~nm} /$ pixel; samples were prepared in a ratio of a 10:1, b 2:1, and c 1:1, respectively

enhancement radius, which is usually estimated to be 6$15 \mathrm{~nm}$ (if the tip radius is $20-50 \mathrm{~nm}$ and the tip-sample distance is $1-2 \mathrm{~nm}[36]$ ), being significantly smaller than the 
pixel size in Fig. 5b. When the pixel size used is larger than $15 \mathrm{~nm}$, the area between two pixels is not being scanned completely by the TERS tip. In other words, the signal only comes from $\mathrm{a} \leq 15 \mathrm{~nm}$ radius below the TERS tip. Therefore, a pixel size larger than $15 \mathrm{~nm}$ can introduce errors in defining domain sizes. This is reflected in our TERS maps, where the features recorded with a coarser pixel size (Fig. 5b) are not reproduced in Fig. 5d. Additionally, along the gaps between gold grains, TERS shows a higher relative peak ratio for OPE. We interpret this to be due to a preferential adsorption of one of the two species, OPE in this case, and also its preferred molecular orientation in these gaps. These edges of gold grains thus show unique features. This is further confirmed by zooming into the same area with a $512 \mathrm{~nm}$ map $(32 \times 32$ points, Fig. 5f), where the Raman spectra of the gap show evidently higher absolute intensity in the TERS map (see ESM Fig. 3c).

The segregation effect of mixing OPE and $\mathrm{PhS}$ was also explored and compared with samples prepared with different molar ratios, 10:1, 2:1, and 1:1, respectively, in solution. TERS peak ratio maps were measured with $8 \mathrm{~nm} /$ pixel. Figure 6a shows islands containing predominantly $\mathrm{PhS}$ with a size around $50 \times 50 \mathrm{~nm}^{2}$ (dark blue). The light blue pixels imply that in these areas some OPE is also present. A larger area map $\left(512 \times 512 \mathrm{~nm}^{2}\right)$ of the same sample is shown in ESM Fig. S4a, which confirms this. Figure $6 \mathrm{~b}$ was acquired in an area of $250 \times 250 \mathrm{~nm}^{2}$, the essentially pure island continuously spans over a size of $80 \times 160 \mathrm{~nm}^{2}$, while there is some OPE distributed around the $\mathrm{PhS}$ domain. The segregation is very strong and agrees well with a TERS map (measured with $2 \mathrm{~nm} /$ pixel) that was acquired with the same ratio (ESM Fig. S4b), where the TERS tip landed inside a $\mathrm{PhS}$ domain, which was wider than $128 \mathrm{~nm}$. The binary SAM prepared with a ratio of 1:1 measured with $8 \mathrm{~nm} /$ pixel also exhibits an essentially pure $\mathrm{PhS}$ island larger than $24 \times 80 \mathrm{~nm}^{2}$ on the bottom of the map, while an OPE domain is located on the left side of the map (depicted in red).

Because TERS mapping yields many data points, it is conceivable to use it to answer whether the molar mixing ratio of thiols in solution reflects the fraction of adsorbed thiols on the surface. Figure S5 in the ESM shows the histograms of the TERS peak ratio for samples prepared with different thiol molar ratios. The total number of data points in each map was 4096, 1024, and 4096, respectively. Histograms (see ESM) show that samples prepared with a ratio of 10:1 and 2:1 exhibit a large fraction of pure $\mathrm{PhS}$ pixels (the peak at $1129 \mathrm{~cm}^{-1}$ has very low intensity, so the ratio is close to 0 ), while samples prepared with a ratio of 1:1 yield significantly more points with a peak ratio larger than 1.5 , which implies that OPE was predominantly adsorbed on the sample surface. These three maps give a consistent trend between the amount of the adsorbed molecules and the molar fraction in a solution, although a complete quantitative agreement was not reached.

\section{Conclusions}

TERS, a nanospectroscopic method, provides a powerful way for spatially resolved $(\approx 10 \mathrm{~nm})$ chemical analysis of surface adsorbates, in cases where STM is "blind." We showed that full spectroscopic mapping of mixed thiol SAMs is possible via TERS and that ratios of marker peaks can be used to discern segregation within the monolayer. If the molecular domains are larger than $15 \mathrm{~nm}$, TERS can give a reliable domain size by using a pixel size in the range of a few nanometers. Histograms generated from many data points were also compared, giving complementary insight about the composition of the SAMs. This is the first time that maps with full chemical information have demonstrated phase segregation in coadsorbed thiols.

Acknowledgments This work was financially supported by the SALSA program at Humboldt University, Berlin, and by the EMRP NEW02 project "Metrology for Raman spectroscopy." The EMRP is jointly funded by the participating countries within EURAMET and the European Union. We would like to thank Lothar Opilik and Jacek Szczerbiński for helpful discussions.

\section{References}

1. Whitesides GM, Kriebel JK, Love JC (2005) Molecular engineering of surfaces using self-assembled monolayers. Sci Prog 88(1): $17-48$

2. Love JC, Estroff LA, Kriebel JK et al (2005) Self-assembled monolayers of thiolates on metals as a form of nanotechnology. Chem Rev 105:1103-1170

3. Thuo MM, Reus WF, Nijhuis CA, Barber JR, Kim C, Schulz MD, Whitesides GM (2011) Odd-even effects in charge transport across self-assembled monolayers. J Am Chem Soc 133(9):2962-2975

4. Stewart A, Zheng S, McCourt MR, Bell SEJ (2012) Controlling assembly of mixed thiol monolayers on silver nanoparticles to tune their surface properties. ACS Nano 6:3718-3726

5. Wu K-Y, Yu S-Y, Tao Y-T (2009) Continuous modulation of electrode work function with mixed self-assembled monolayers and its effect in charge injection. Langmuir 25:6232-6238

6. Kim J, Rim YS, Liu Y et al (2014) Interface control in organic electronics using mixed monolayers of carboranethiol isomers. Nano Lett 14:2946-2951

7. Hung A, Mwenifumbo $S$, Mager $M$ et al (2011) Ordering surfaces on the nanoscale: implications for protein adsorption. J Am Chem Soc 133:1438-1450

8. Hobara D, Imabayashi S-I, Kakiuchi T (2002) Preferential adsorption of horse heart cytochrome con nanometer-scale domains of a phase-separated binary self-assembled monolayer of 3mercaptopropionic acid and 1-hexadecanethiol on $\mathrm{Au}(111)$. Nano Lett 2:1021-1025

9. Smith RK, Reed SM, Lewis PA et al (2001) Phase separation within a binary self-assembled monolayer on $\mathrm{Au}\{111\}$ driven by an amide-containing alkanethiol. J Phys Chem B 105:1119-1122

10. Qi Y, Liu X, Hendriksen BLM et al (2010) Influence of molecular ordering on electrical and friction properties of $\omega$-(trans-4stilbene)alkylthiol self-assembled monolayers on $\mathrm{Au}$ (111). Langmuir 26:16522-16528 
11. Stranick SJ, Atre SV, Parikh AN, Wood MC, Allara DL, Winograd N, Weiss PS (1997) Nanometer-scale phase separation in mixed composition self-assembled monolayers. 1-6

12. Hayes WA, Kim H, Yue X, Perry SS, Shannon C (1997) Nanometer-scale patterning of surfaces using self-assembly chemistry. 2. Preparation, characterization, and electrochemical behavior of two-component organothiol monolayers on gold surfaces. Langmuir 13:2511-2518

13. Poirier GE (1997) Characterization of organosulfur molecular monolayers on $\mathrm{Au}(111)$ using scanning tunneling microscopy. Chem Rev 97:1117-1127

14. Schaefer AH, Seidel C, Chi L, Fuchs H (1998) STM investigations of thiol self-assembled monolayers. Adv Mater 10:839-842

15. Stadler J, Schmid T, Opilik L, Kuhn P, Dittrich PS, Zenobi R (2011) Tip-enhanced Raman spectroscopic imaging of patterned thiol monolayers. Beilstein J Nanotechnol 2:509

16. Wang DW, Tian F, Lu JG (2002) Characterization of self-assembled alkanethiol monolayers using a low-current scanning tunneling microscope. J Vac Sci Technol B 20:60-64

17. Mannsfeld SCB, Canzler TW, Fritz T et al (2002) The structure of [4-(phenylazo)phenoxy] hexane-1-thiol self-assembled monolayers on Au(111). J Phys Chem B 106:2255-2260

18. Donten ML, Królikowska A, Bukowska J (2009) Structure and composition of binary monolayers self-assembled from sodium 2mercaptoetanosulfonate and mercaptoundecanol mixed solutions on silver and gold supports. Phys Chem Chem Phys 11:3390-3400

19. Centrone A, Hu Y, Jackson AM et al (2007) Phase separation on mixed-monolayer-protected metal nanoparticles: a study by infrared spectroscopy and scanning tunneling microscopy. Small 3:814 817

20. Gentilini C, Franchi P, Mileo E et al (2009) Formation of patches on 3D SAMs driven by thiols with immiscible chains observed by ESR spectroscopy. Angew Chem 121:3106-3110

21. Bain CD, Whitesides GM (1998) Science 240:6243

22. Harkness KM, Balinski A, McLean JA, Cliffel DE (2011) Nanoscale phase segregation of mixed thiolates on gold nanoparticles. Angew Chem Int Ed 50:10554-10559

23. Lipiec E, Sekine R, Bielecki J et al (2013) Molecular characterization of DNA double strand breaks with tip-enhanced Raman scattering. Angew Chem Int Ed 53:169-172

24. Najjar S, Talaga D, Schué L et al (2014) Tip-enhanced Raman spectroscopy of combed double-stranded DNA bundles. J Phys Chem C 118:1174-1181

25. Stadler J, Schmid T, Zenobi R (2010) Nanoscale chemical imaging using top-illumination tip-enhanced Raman spectroscopy. Nano Lett 10:4514-4520

26. Hayazawa N, Inouye Y, Sekkat Z, Kawata S (2000) Metallized tip amplification of near-field. Raman Scattering 183(1-4):333-336

27. Anderson MS (2000) Locally enhanced Raman spectroscopy with an atomic force microscope. Appl Phys Lett 76:3130-3132

28. Stöckle RM, Suh YD, Deckert V, Zenobi R (2000) Nanoscale chemical analysis by tip-enhanced. Raman Spectroscopy 318(13):131-136

29. Liu Z, Wang X, Dai K et al (2009) Tip-enhanced Raman spectroscopy for investigating adsorbed nonresonant molecules on singlecrystal surfaces: tip regeneration, probe molecule, and enhancement effect. J Raman Spectrosc 40:1400-1406

30. Zheng Z, Opilik L, Schiffmann F et al (2014) Synthesis of twodimensional analogues of copolymers by site-to-site transmetalation of organometallic monolayer sheets. J Am Chem Soc 136:6103-6110

31. Sonntag MD, Klingsporn JM, Garibay LK et al (2012) Singlemolecule tip-enhanced Raman spectroscopy. J Phys Chem C 116: $478-483$
32. Steidtner J, Pettinger B (2008) Tip-enhanced Raman spectroscopy and microscopy on single dye molecules with $15 \mathrm{~nm}$ resolution. Phys Rev Lett 100:236101

33. Zhang R, Zhang Y, Dong ZC et al (2013) Chemical mapping of a single molecule by plasmon-enhanced Raman scattering. Nature 498:82-86

34. Picardi G, Chaigneau M, Ossikovski R et al (2009) Tip enhanced Raman spectroscopy on azobenzene thiol self-assembled monolayers on $\mathrm{Au}(111)$. J Raman Spectrosc 40:1407-1412

35. Picardi G, Królikowska A, Yasukuni R, Chaigneau M, Escude M, Mourier V, Licitra C, Ossikovski R (2014) Exchange of methyl- and azobenzene-terminated alkanethiols on polycrystalline gold studied by tip-enhanced Raman mapping. ChemPhysChem 15:276-282

36. Horimoto NN, Tomizawa S, Fujita Y, Kajimoto S, Fukumura H (2014) Nano-scale characterization of binary self-assembled monolayers under an ambient condition with STM and TERS. Chem Commun 50:9862-9864

37. Munakata H, Kuwabata S, Yoshihisa O, Yoneyama H (2001) Spatial distribution of domains in binary self-assembled monolayers of thiols having different lengths. J Electroanal Chem 496(1-2):29-36

38. Stranick SJ, Parikh AN, Tao YT, Allara DL, Weiss PS (1994) Phase separation of mixed-composition self-assembled monolayers into nanometer scale molecular domains. J Phys Chem 98(31):7636-7646

39. Stadler J, Schmid T, Zenobi R (2010) Nanoscale chemical imaging using top-illumination tip-enhanced Raman spectroscopy. Nano Lett 10:4514-4520

40. Blum C, Schmid T, Opilik L et al (2012) Missing amide I mode in gap-mode tip-enhanced Raman spectra of proteins. J Phys Chem C 116:23061-23066

41. Schmid T, Opilik L, Blum C, Zenobi R (2013) Nanoscale chemical imaging using tip-enhanced Raman spectroscopy: a critical review. Angew Chem Int Ed 52:5940-5954

42. Yu J-J, Tan YH, Li X et al (2006) A nanoengineering approach to regulate the lateral heterogeneity of self-assembled monolayers. J Am Chem Soc 128:11574-11581

43. Liu Z, Wang X, Dai K et al (2009) Tip-enhanced Raman spectroscopy for investigating adsorbed nonresonant molecules on singlecrystal surfaces: tip regeneration, probe molecule, and enhancement effect. J Raman Spectrosc 40:1400-1406

44. Edinger K, Goelzhaeuser A, Demota K, Woell C, Grunze M (1993) Formation of self-assembled monolayers of n-alkanethiols on gold: a scanning tunneling microscopy study on the modification of substrate morphology. Langmuir 9(1):4-8

45. Chernia Z, Livneh T, Pri-Bar I, Koresh JE (2001) Mode assignment for linear phenyl acetylene sequence: phenylacetylene, diphenylacetylene and 1,4-di(phenylethynyl)benzene. Vib Spectrosc 25:119-131

46. Joo S-W, Kim K (2004) Adsorption of phenylacetylene on gold nanoparticle surfaces investigated by surface-enhanced Raman scattering. J Raman Spectrosc 35:549-554

47. Liu Z, Wang X, Dai K et al (2009) Tip-enhanced Raman spectroscopy for investigating adsorbed nonresonant molecules on singlecrystal surfaces: tip regeneration, probe molecule, and enhancement effect. J Raman Spectrosc 40:1400-1406

48. Chaigneau M, Picardi G, Ossikovski R (2011) Molecular arrangement in self-assembled azobenzene-containing thiol monolayers at the individual domain level studied through polarized near-field Raman spectroscopy. IJMS 12:1245-1258

49. Opilik L, Bauer T, Schmid T et al (2011) Nanoscale chemical imaging of segregated lipid domains using tip-enhanced Raman spectroscopy. Phys Chem Chem Phys 13:9978-9981

50. Kudelski A (2010) Raman characterization of monolayers formed from mixtures of sodium 2-mercaptoethanesulfonate and various aromatic mercapto-derivative bases. J Phys Chem B 114:5180 5189 